hep-th/9506???

June 1995

\title{
A Note on the Wodzicki Residue
}

\author{
Thomas Ackermann*
}

Wasserwerkstr. 37, D-68309 Mannheim, F.R.G.

\begin{abstract}
In this note we explain the relationship of the Wodzicki residue of (certain powers of) an elliptic differential operator $P$ acting on sections of a complex vector bundle $E$ over a closed compact manifold $M$ and the asymptotic expansion of the trace of the corresponding heat operator $e^{-t P}$. In the special case of a generalized laplacian $\triangle$ and $\operatorname{dim} M>2$, we thereby obtain a simple proof of the fact already shown in $[\mathrm{KW}]$, that the Wodzicki residue $\operatorname{res}\left(\triangle^{-\frac{n}{2}+1}\right)$ is the integral of the second coefficient of the heat kernel expansion of $\triangle$ up to a proportional factor.
\end{abstract}

Keywords: non-commutative residue, heat kernel expansion 1991 MSC: 47F05, 53B50, 58G11

* e-mail: ackerm@euler.math.uni-mannheim.de 


\section{Introduction}

In $[\mathrm{KW}]$ it was shown that the Wodzicki residue $\operatorname{res}\left(\triangle^{-\frac{n}{2}+1}\right)$ of a generalized laplacian $\triangle$ on a complex vectorbundle $E$ over a closed compact manifold $M$, $\operatorname{dim} M=n>2$, is the integral of the second coefficient of the heat kernel expansion of $\triangle$ up to a proportional factor. This observation reflects a more general property which we explain in this note, namly that the Wodzicki residue of (certain powers of) an elliptic differential operator $P$ on $E$ can be related with the coefficients in the asymptotic expansion of the trace of the corresponding heat operator $e^{-t P}$. Even if this might be well-known by mathematicians working in the field - our simple proof relies on two results made by Wodzicki [W] and Gilkey [Gi] we think it worth to restate it because of the growing importance of the Wodzicki residue for non commutative geometry, for example to incorporate gravity in the Connes-Lott model (cf. [CFF]). By the way we correct an error term in $[\mathrm{KW}]$.

\section{Computing $\operatorname{res}\left(P^{-\left(\frac{n-k}{d}\right)}\right.$}

Let $E$ be a finite dimensional complex vector bundle over a closed compact manifold $M$ of dimension $n$. Recall that the non-commutative residue of a pseudo-differential operator $P \in \Psi \operatorname{DO}(E)$ can be defined by

$$
\operatorname{res}(P):=(2 \pi)^{-n} \int_{S^{*} M} \operatorname{tr}\left(\sigma_{-n}^{P}(x, \xi)\right) d x d \xi
$$

where $S^{*} M \subset T^{*} M$ denotes the co-sphere bundle on $M$ and $\sigma_{-n}^{P}$ is the component of order $-n$ of the complete symbol $\sigma^{P}:=\sum_{i} \sigma_{i}^{P}$ of $P$, cf [W]. In his thesis, Wodzicki has shown that the linear functional res: $\Psi \mathrm{DO}(E, F) \rightarrow \mathbb{C}$ is in fact the unique trace (up to multiplication by constants) on the algebra of pseudo-differential operators $\Psi \mathrm{DO}(E)$.

Now let $P \in \Psi D O(E)$ be elliptic with ord $P=d>0$. It is well-known (cf. [Gi]) that its zeta function $\zeta(P, s)$ is a holomorphic function on the half-plane $\operatorname{Re} s>n / d$ which continues analytically to a meromorphic function on $\mathbb{C}$ with simple poles at 
$\left\{\frac{(n-k)}{d} \mid k \in \mathbb{N} \backslash\{n\}\right\}$. For $n-k>0$ with $k \in \mathbb{N}$ we have the following identities:

$$
\begin{aligned}
\operatorname{res}\left(P^{-\left(\frac{n-k}{d}\right)}\right) & =d \cdot \operatorname{Res}_{s=\frac{n-k}{d}} \zeta(P, s) \\
R e s_{s=\frac{n-k}{d}} \zeta(P, s) & =a_{k}(P) \cdot \Gamma\left(\frac{n-k}{d}\right)^{-1} .
\end{aligned}
$$

Here $\Gamma$ is the gamma function and $a_{k}(P)$ denotes the the coefficient of $t^{\frac{k-n}{d}}$ in the asymptotic expansion of $\operatorname{Tr}_{L^{2}} e^{-t P}$. The first equality was shown in [W], whereas (2.3) is a consequence of the Mellin transform which relates the zeta function and the heat equation and was already proven by Gilkey in [Gi]. Consequently we obtain

$$
a_{k}(P)=d^{-1} \cdot \Gamma\left(\frac{n-k}{d}\right) \cdot \operatorname{res}\left(P^{-\left(\frac{n-k}{d}\right)}\right) .
$$

Now suppose $P=\triangle$ is a generalized laplacian and $k=2$. Then $a_{2}(\triangle)=$ $(4 \pi)^{-\frac{n}{2}} \phi_{2}(\triangle)$ where $\phi_{2}(\triangle)$ denotes the integral over the diagonal part of the second coefficient of the heat kernel expansion of $\triangle$. By using the well-known identity $\Gamma(z+1)=z \Gamma(z)$ it is easily verified that

$$
(n-2) \phi_{2}(\triangle)=(4 \pi)^{\frac{n}{2}} \cdot \Gamma\left(\frac{n}{2}\right) \cdot \operatorname{res}\left(\triangle^{-\frac{n}{2}+1}\right)
$$

for $n>2$. Note, that in the above mentioned reference $[\mathrm{KW}]$ the Wodzicki residue was defined by $\widetilde{r e s}(P):=\frac{\Gamma\left(\frac{n}{2}\right)}{2 \pi^{\frac{n}{2}}} \int_{S^{*} M} \operatorname{tr}\left(\sigma_{-n}^{P}(x, \xi)\right) d x d \xi$, using $\operatorname{vol}\left(S^{n-1}\right)^{-1}=\frac{\Gamma\left(\frac{n}{2}\right)}{2 \pi^{\frac{n}{2}}}$ as a nomalizing factor. Thus, equation (2.5) can be equivalently expressed as $\widetilde{r e s}\left(\triangle^{-\frac{n}{2}+1}\right)=\frac{n-2}{2} \phi_{2}(\triangle)$. Therefore their proportional factor in equation (4.10) is not correct.

We conclude in exploiting the result we have in hand in the case of a Clifford module $E=\mathcal{E}$ over a four-dimensional closed compact Riemannian manifold $M$ and $\triangle$ being the square of a Dirac operator $D$ defined by a Clifford connection. Then equation (2.5) together with the well-known observation $\phi_{2}\left(D^{2}\right) \sim \int_{M} * r_{M}$, where $*$ is the Hodge star corresponding to the Riemannian metric and $r_{M}$ denotes the scalar curvature of $M$, yields $\operatorname{res}\left(D^{-2}\right) \sim \int_{M} * r_{M}$. Thus, by the above derivation of (2.5), the fact - first announced by Connes [C] and shown by Kastler [K] using the symbol calculus - that the non-commutative residue of the inverse square of the Dirac operator is proportional to the Einstein-Hilbert action of general relativity, is almost obvious.

Note: After completion of this work we have been told that this result was independently recognized by M. Walze [Wa]. 


\section{References}

[C] A.Connes, Non-commutative geometry and physics, IHES preprint (1993)

[CFF $] \quad$ A.H.Chamseddine, G.Felder, J.Fröhlich, Gravity in non-commutative geometry, Com. Math. Phys 155 (1993), 205-217

[Gi] P.G.Gilkey, Invariance theory, the heat equation, and the Atiyah-Singer index theorem, Publish or Perish (1984)

[K] D.Kastler, The Dirac operator and gravitation, Com. Math. Phys. 166(1995)

[KW] W.Kalau, M.Walze, Gravity, non-commutative geometry and the Wodzicki residue, to appear in Journ. of Geometry and Physics

[W] M.Wodzicki, Non-commutative residue I, LNM 1289 (1987), 320-399

[Wa] M.Walze, Nicht-kommutative Geometrie und Gravitation, thesis, Universität Mainz, to appear 